\title{
Storing and Retrieving Documents in a Shared Workspace: Experiences from the Political Administration
}

\author{
Volker Wulf \\ Institute for Computer Science III \\ University of Bonn \\ Römerstr. 164, D-53117 Bonn, Germany \\ Email: volker@informatik.uni-bonn.de
}

\begin{abstract}
In the following we will describe experiences made when introducing a shared workspace in a German federal ministry to support the cooperative typing of documents. It will be shown how the shared workspace improved the typing process, how storing and retrieval conventions evolved during a two-year period, and which technical improvements of the system functionality have been required by the users to ease document retrieval. Finally, it will be discussed which organizational and technical factors support the establishment of common storing and retrieving conventions in shared workspaces.
\end{abstract}

KEYWORDS Groupware, Shared Workspaces, Classification Scheme, Document Retrieval, Field Study

\section{INTRODUCTION}

As the production of documents is increasingly based on the application of personal computers, storage and retrieval of electronic documents are an important research topic. Nevertheless, studies on storing and retrieval habits of office employees have focused up to now mainly on the handling of paper documents. There exist only few empirical studies dealing with the file organization in the electronic office (cf. Barreau and Nardi 1995; Rao et al. 1994). These studies look at the organization of electronic documents from a single user perspective. Since the production of documents gets more of a cooperative activity with networked computers, additional research on the organization of electronic documents in shared workspaces is required.

From a methodological point of view, most of the studies on the organization of electronic and physical files have taken a static perspective in the sense that the data were gathered at just one point in time (e.g. Malone 1983; Suchman and Wynn 1984, Barreau 1995; Nardi, Anderson and Erickson 1994). Since the organization of documents - especially in collaborative environments might evolve considerable during time, a more long term research approach could lead to interesting results. The period following the introduction of a new computer system seems to be an especially interesting time span. Here we assume to observe how conventions for the organization of electronic documents are established based on prior experiences and how they evolve later on.

In the following, we will report on the evolution of storing and retrieving habits in a shared workspace. The shared workspace was introduced to ease the cooperative production of typed documents in a German federal ministry. Based on the experiences made during a two years span, we will draw some conclusions on how to support the establishment of storing and retrieving conventions in shared workspaces. 


\section{STORING AND RETRIEVING IN INDIVIDUAL WORKSPACES}

As the focus of this study has been influenced by the results of already existing research work, I will give a brief survey on its results. Interviewing ten office workers on the physical organization of their desks, Malone (1983) distinguishes files and piles as mayor units of desk organization. While in files the individual documents are titled and ordered, in piles there is not any structure of the containing documents. Piles themselves are not named, either. The organization of the documents seems to be related to their contend.

As the result of an empirical study on 15 experienced Macintosh users of different professional background, Nardi, Anderson and Ericson (1994) distinguish three types of information represented on electronic documents: "ephemeral", "working", and "archived". While ephemeral information is needed only for a short period of time (e.g.: "to do"-lists, memos, ...), working information is created by the users when carrying out their actual tasks. It has a typical shelf time of weeks or months. By contrary, archived information is only indirectly relevant to users' actual work. It represents completed work and has a shelf time of months or years. The authors found out that different types of information are stored in different ways by the users. While ephemeral information is kept rather in "electronic piles" either unstructured on the desktop or loosely filed at the top directory level, working and archived information is more systematically filed.

Barreau (1995) has interviewed seven managers of a large information management company to find out how they store and retrieve electronic documents. In comparing the classification schemes applied by the interviewees in her study with those of an earlier study carried out by Kwasnik (1989) on the organization of paper documents, she found that "...(m)any of the same types of documents were identified, and many of the same dimensions were used in making classification decisions" (p. 336). Nevertheless, comparing the classification schemes of different individuals she found "completely different rules for classifying documents based upon the level of granularity required to support the workload" (p. 337). Malone (1983) had already pointed to the fact that different office workers showed great variations in how precisely they organized their offices. Performing interviews about the work practice of 16 office workers of a large office equipment manufacturer, Suchman and Wynn (1984) have come to a similar result. They state that the most efficient and satisfactory pattern of desktop organization are discovered rather than prescribed (cf. Suchman and Wynn 1984, p. 150). Morcover, Barreau (1995) stated that classification "(r)ules that are applied for a period of time to reflect the priorities of the moment may soon be abandoned or forgotten..." (p. 337).

Concerning the methods to search for an electronic document, Barreau and Nardi (1995) distinguish between "location based" and "logical" finding. In location based finding a user makes a guess for the folder or directory where he thinks a document might be located, goes to that location, and browses the documents located there. This process is iterated if needed. In logical finding the user specifies an inquiry by filling in attribute values or keywords related to the document he is searching for. As a result he gets listed all the documents which correspond to the inquiry. While location based finding is similar to the searching process for paper documents, logical finding can be just applied for electronic documents. Both of the studies, which look at the users' habits to search for electronic documents indicate an overwhelming preference for location based search. The authors attribute this fact to the higher level of control the users exercise over the location based search process and problems in remembering the right attribute values (e.g. the document's name) of the desired document (cf. Barreau and Nardi 1995, pp. 40).

Looking at search processes for paper documents on colleagues' desks, Suchman and Wynn (1984) point to problems resulting from the fact that the way papers are organized on one person's desk is not easily apparent to other workers. Therefore, Barreau and Nardi (1995) assume that for search outside of the individual desk, tools for logical finding might be more appropriate than for searching on the individual desk.

Moreover, empirical studies have indicated that the allocation of documents on the desk has a critical reminding function. Malone (1983) has already hinted to this fact when looking from an individual perspective at the physical organization of papers on desks. Suchman and Wynn (1984, pp. 147) add that the localisation of documents on an employee's desk often indicates the state of processing to his colleagues. The reminding function of the allocation of documents has also been observed in the studies on the organization of electronic documents (cf. Barreau and Nardi 1995).

\section{THE RESEARCH SETTING}

This research took place in the context of the POLITeam project. Within the POLITeam project a groupware application is developed in an evolutionary 
and participative way. The first system version is generated by configuring the commercial product LinkWorks by Digital (POLITeam I). Based on the experiences gained by introducing the first system version in three different fields of application, we develop advanced versions of the system. The functionality mainly consists of an electronic circulation folder, shared workspaces, and an event notification service. Looking at the means to organize files, the system is based on the desktop metaphor and offers an electronic folder hierarchy. These folders can be either used only on one's personal desktop or they can be shared with other users (cf. Klöckner et al. 1995, Mambrey, Mark and PankokeBabatz 1996).

In the following we will report about experiences made in one of the three different fields of application of the POLITeam project. The field of application is a section of a federal ministry. The section consists of about five employees and one section manager. The work of the section is supported by typists and a worker in the registrar. The typists belong to the central typing pool whose offices are located in another part of the building. The registrar is allocated on another floor of the same building. Before introducing the POLITeam system, the typing pool was equipped with net-worked PCs, using a common server to store the text documents. The employees of the operating section and in the registrar were not equipped with computers for their daily work.

This report is based on results of initial interviews with the users concerning their work practice before the introduction of POLITeam, on interviews carried out after the introduction of POLITeam, on experiences gained by providing regular system support after the introduction of POLITeam, and on an evaluation of several workshops held in this field of application. Within the first workshop, we presented the prototypical configuration to the users (workshop A), half a year after the introduction of POLITeam I we held a workshop to discuss problems the users were facing when applying POLITeam I (workshop B), about another half a year later we presented the POLITeam II version to the users (workshop C) and the most recent workshop was held half a year after the introduction of POLITeam II to represent some features of the new system version and to discuss problems of use (workshop D). In all of the workshops employees of the section of the ministry, the section manager, employees of the typing pool, the head of the ministry's computer department and members of the project-team working as system support and as designers participated.

\section{STORING AND RETRIEVING DOCUMENTS BEFORE THE INTRODUCTION OF POLITEAM}

One of the main tasks of the ministry's section observed, consists of the development of concepts for a certain field of politics. Some of these concepts are developed just within the section. Others are worked out in cooperation with other sections of the ministry. These concepts (e.g. manuscripts for speeches of the minister, answers to inquiries from the parliament, or answers to letters sent by citizens) are documented on paper and provided by means of circulation folders for the upper level of the ministry's hierarchy. All the paper documents, for internal as well as for external use, are typed.

Before the introduction of POLITeam all type work for the ministry was carried out by the central typing pool. Therefore, members of the section sent handwritten texts or tapes with voice recording to the typing pool. After typing, the texts were sent back to the sender who checked the document. If he found a mistake, he had to send it back to the typist who did the correction. This version of the document was finally given to the section manager who was responsible to process it and who might have asked for further modifications. As the transportation of the material between the employees of the section and the typists was normally done by internal messengers and as the typists had to schedule their different tasks, it normally took three to four days for the typing of the first version of a document. Thus if a task was very urgent, the section members used to bring their manuscripts to the typists personally and asked for a preferential treatment. But even this procedure took time, especially if the typed version contained mistakes. These problems were the reason why the introduction of POLITeam led to a change in the typing process. This process innovation influenced the storing conventions for typed documents strongly.

Before the introduction of POLITeam typed documents were stored twice in the ministry, electronically and physically. These stores served different purposes and thus were built up in a very different way. The - still existing - physical store of the whole ministry is handled by the employees of the registrar. Within the registrar individual paper documents are filed in folders. A folder contains all paper documents related to a specific task of the ministry. All the tasks of the ministry can be classified according to a ministrywide classification scheme. The employees in the 
registrar create folders for all the tasks performed in the ministry as soon as they receive paper documents related to them. For instance, if tasks are initiated by an incoming mail these letters are normally routed via the registrar before they reach the individual sections. Thus the registrar classifies it, looks whether there exists already a corresponding folder (ongoing task), or creates a new folder (new task). A new folder receives an index according to the task classification scheme of the ministry. The registrar uses this index to store and retrieve the folders because their physical placement in the registrar is related to the index.

If the ministry is working on certain tasks the corresponding folders are taken out of the registrar to the sections which are responsible for them. For instance, in case of a task created by an incoming letter, the registrar will send the newly created folder to the organizational unit responsible for its performance. Members of a specific operative section responsible for the task will hold the folder as long as they work on it. Though members of a section are not allowed to store folders in their offices for a longer period of time, the arrangement of different piles of folders in certain areas of their offices is used by them to remind themselves of the corresponding tasks. To remind section members of tasks which have to be performed in the future, the employees of the registrar provide a corresponding service. They keep a list of these tasks and send the corresponding folders at the predefined dates to the sections. The physical reception of a folder reminds the members of the operation sections of the tasks. Thus, folders containing all the paper documents related to a especial task are the basic units for storing and retrieving physical documents in the ministry.

By contrary the storage of electronic documents was based on individual documents. Before the introduction of the POLITeam system, the typing pool was the only organizational unit which stored documents electronically. The typing pool had created directories for each section of the ministry, containing subfolder for each member of the sections. Within these subfolders all the documents which any typist had written for a certain client were stored. On the third level of the folderhierarchy, the typists distinguished among five different types of documents to be stored in the same subfolder. Thus, if a client would ask for corrections on a certain type of document, any typist knew in which subfolder to search. To ease finding within the subfolders, all typists used a common convention for the naming of documents. Using the DOS operation system, they were restricted to the use of eight symbols to name documents. The first five symbols of the documents' name were used to indicate the date of a document's first creation, expressing day, month and year in reverse order. Thus, it was possible to search within the subfolders using a temporal structure. This naming and storing conventions were convenient for the search needs of the typing pool because they eased location based finding of individual documents. Nevertheless they were incompatible with the conventions for storing paper documents. This fact became obvious when other parts of the ministry started to work electronically, as well.

\section{STORING AND RETRIEVING DOCUMENTS AFTER THE INTRODUCTION OF POLITEAM}

After the introduction of the POLITeam system the mode to produce typed documents has changed. It was decided to create a shared workspace consisting of a folder which could be accessed by the operative section and the typing pool. Within this folder all documents were stored which the typing pool produced for the members of the section. The folder contained subfolders to store documents produced for each member of the section.

The introduction of folders shared between the section and the typing pool allowed new modes for the division of labour. Being equipped with a word processing tool and having the chance to gain access to typed documents, the members of the section could decide flexibly what to type themselves and when to ask the typing pool. Each of them developed in the following an individual strategy. Moreover, the section manager who checked all the documents leaving the section typed his corrections himself by accessing the shared folder. Though there was a change in the way typed documents were produced within the section, the handling of typed documents outside of the section did not change. As the other sections, the hierarchy of the ministry, and the registrar were not yet equipped with computers, all documents got printed out and left the section in traditional circulation folders.

When POLITeam I was introduced, the shared workspace consisted of a two level hierarchy of folders. In the folder shared between section and typing pool there were subfolders containing the documents for each member of the section. To maintain their location based search strategy, the typists changed the naming convention by starting the name of each document with a letter indicating the document type and continuing with numbers, indicating the date of a document's creation. The access to the shared folder was implemented in a way 
that the head of the section and the typists could read and write all documents in the whole folder while the other members of the section could just access documents in their subfolders.

During workshop B on problems of use, half a year after the introduction of POLITeam I, the members of the operative section expressed their dissatisfaction concerning these patterns of naming and storing. When searching for documents they do not think of document types, but of their tasks. Working on a task means to create various documents of different types. Thus, the mode of storing and naming did not reflect their needs for the support of a location based finding strategy. Discussing this topic, a conflict of interest between the section and the typing pool became manifest. As both organizational units classified the documents in a different way a single location based organization of the documents would not satisfy both sides. In this situation the members of the POLITeam project proposed different solutions. First, one member of the POLITeam project suggested to keep the naming and storing system like it was, but to tell the members of the section to display the documents in the temporal order of their creation. She assumed that such a display would come close to a taskoriented structure of storage, because documents concerning the same task would be created around the same time. This proposal was perceived positively within the workshop but it was not possible to implement because POLITeam I just allowed to display documents according to the time of their last modification. The members of the section felt that such a display would not be sufficiently task-oriented. Thus, this compromise which had allowed to generate two different patterns to organize documents within a folder could not be implemented due to a lack of flexibility of the interface display function.

The solution agreed on finally in the workshop, was to allow the members of the section to introduce a third level in the folder-hierarchy, where documents could be allocated according to the section member's task they belong to. The naming of the documents was kept like it was. Thus, the classification scheme of the operative section became the base for the storage of the documents. Nevertheless, it turned out that the compromise found in the workshop was not put widely into practice.

Another problem became obvious after the introduction of the POLITeam I version. As the cooperation between the section and the typing pool was based on shared folders, the typed documents were neither sent electronically nor physically from the typists to the section members. Before the introduction of POLITeam, the reception of a typed document informed the members of the section that the typing process was finished and reminded them of the corresponding task. After the introduction of POLITeam the typists stored newly typed documents in the shared subfolder. Thus, the members of the section were not notified explicitly, they had to look themselves in their folder to check whether a document has been typed. Therefore, the members of the section asked in the workshop B for technical functions which make typed documents visible to them. They wanted to see by means of the colour of an icon, whether the typists have finished working on the corresponding document. Moreover, they wanted to recognize newly created files by a different colour of icon. As POLITeam I did not offer these options, we discussed different intermediate solutions. First of all, it was suggested that the typists should send a mail whenever they finished working on a document. The typists found it too labourintensive and the members of the section feared the flood of incoming mails. As an intermediate solution, they agreed to use the rudiment features of the already existing event service.

About 12 months after the introduction of the POLITeam I version, we equipped the users with a new system version (POLITeam II). In this version we had realized those user requirements which where rather simple to implement within the base system. Concerning the storage and retrieval of documents the following features were most relevant. Location based finding got supported by the introduction of a viewer which allows to scan into individual documents without opening the corresponding application. Moreover, a function was implemented which allowed to select between different display-modes of documents within a folder. For instance, the users could organize documents according to their names, their date of creation, or their index which has become an additional attribute of the electronic documents. Thus, within a folder the individual users could select among different modes to allocate documents.

Furthermore, the POLITeam II version offered a tool for logical finding of documents. Though the LinkWorks product version contained already such a search tool, it had been deactivated when configuring the POLITeam I version. This was due to the fact that the original search tool allowed to find any document within the system independent of the location where it was stored. Thus, users could have retrieved, accessed and modified documents which were allocated on the private desktop of other users. As we assumed that such a search tool would have endangered the acceptance of the whole system, we 
decided to deactivate this feature. For the POLITeam II version we had modified the search tool in such a way that users could only find documents which were accessible on their own desktop (cf. Kahler 1996). In the following we will report on the further development of the file organization in the shared folder and the experiences made with the new features of the POLITeam II version.

Looking at the file organization in the shared workspace about one year after the agreement in workshop B, the majority of the members of the section still worked with a two level folder-hierarchy. Nevertheless, in interviews several of them complained that the amount of documents within their subfolders which made it very troublesome to find any document.

For instance, one member of the section pointed out that she had not used subfolders in her subdirectory because their creation and the classification of documents led to additional work. She appreciated very much the additional features to display documents according to different criteria. This was the only way for her to find documents in her vast subfolder. Nevertheless, after a year of document production her subfolder is meanwhile that crowded with documents that she has real problems in finding them. This is especially the case for older documents which she reuses occasionally. Thus, on the one hand she regreted that she did not build up a third level in the folder hierarchy to ease document-retrieval. On the other hand she avoided the additional workload. Moreover she stated that the classification and archiving of documents is not her job. She told us that she is not familiar with the classification scheme of the ministry and expects support from the registrar concerning the electronic storage of documents.

By contrary, one of her colleagues has developed an own classification scheme by creating task-related subfolders to store documents. When asking the typing pool to get a document typed, she indicates the corresponding subfolder on the manuscript. She finds this organization of documents to be very helpful to support her daily work.

Thus, the members of the section have developed rather individual strategies for the organization of the documents in the folder shared with the typists. Therefore, the storage scheme within this folder became a topic of discussion in workshop D. The manager of the section brought up a controversy. Though he had advocated the compromise negotiated within the workshop B, he was meanwhile of the opinion that there should not be any third level within the folder hierarchy of the shared workspace. He stated that the newly introduced display function should offer sufficient support to search for documents. A further level of the folder hierarchy made it very difficult for him to retrieve documents within the other section member's subfolders. He especially insisted on the fact that the index attribute should be filled in every electronic document. A display of the documents according to this index should be a sufficient support for location based retrieval.

In the following the members of his section who had created a third level in the folder hierarchy explained how helpful such an organization was for their work. A member of the project-team supported them by explaining how a third level of the folder hierarchy together with the newly introduced display function can support location based finding. As the section manager insisted on a standardised file organization, the member of the project-team suggested to allow only subfolders on the third level of the hierarchy, which are created according to the organization-wide classification scheme. After discussing details like which version of the classification scheme should be valid and on which level of granularity the scheme should be applied, the new convention was agreed upon by all the users.

Though the search tool has been introduced in the POLITeam II version the facts presented up to now indicate that most of the documents are still retrieved by location based finding. For instance, one of the section members stated in an intervicw that she uses the search tool less than once a week. She needs it mainly if she cannot remember the folder a document is stored in or if a document has been mistakenly moved into a wrong folder.

In workshop $\mathrm{D}$ the question arose whether it should be possible to apply logical finding to those documents which can not be accessed by location-based finding. The typists were in favour of such an implementation because they had made the experiences that the members of the section did not keep all the relevant documents stored in the shared folder. Thus they would prefer to be able to search on the section member's private desks. Moreover, they are sometimes asked by members of the section to search for a specific document in another section member's shared subfolder because the typists can access the whole shared workspace. Thus, they had to provide manual services to the section which an extended search tool could provide electronically.

By contrary, the members of the section were less enthusiastic about an extended function for logical finding. On the one hand they argued that finding documents on the desktop of a colleague with whom they do not co-operate at all does not make much sense. 
On the other hand they fear that their privacy would get violated if, for instance, somebody of the hierarchy of the ministry could check all documents an employee has created during a certain period of time. Moreover, documents been stored on their private desk are often in an unfinished state and they would not like to allow access to them before they get ready. After discussing this topic for a while a member of the project-team suggested a redesign of the search tool which would allow to differentiate the behaviour of the search tool according to the location a document is stored. Thus, documents stored in certain locations should not be found at all, in other locations the search tool would just display the location of the documents while in other cases the documents could be accessible directly. Such a design of the search tool was thought to be a viable way to extend the search tools scope beyond a user's own desktop.

\section{CONCLUSION}

In the case study reported we saw how storing and retrieval habits on working and archived information evolved after the introduction of groupware. Introducing a groupware system which is based on the desktop metaphor, we observed strong preferences of the users for location based finding in comparison to logical finding. This finding might be due to the fact that the system did not contain a search tool before the POLITeam II version. Nevertheless this finding concerning shared workspaces is consistent with results on single user's workspaces. Thus, it seems to be important to focus research and tool development on location based finding.

Concerning logical finding in groupware, the users require that these tools should be sensitive to the location a document is stored. To avoid information overload and to protect the privacy of certain locations, search-tools should not display all the documents fitting to the specification of an inquiry. Moreover, it seems to be reasonable that search tools provide different mechanisms to control access to documents which are allocated outside of the searchers own workspace. This leads to a different design of search tools for collaborative environments in comparison to those for single user systems (cf. Kahler 1996, Wulf 1997).

Looking at the establishment of a classification scheme for the location based storage of electronic documents, we have experienced a long lasting negotiation process. Within this process, we are facing two different problems. On the one hand two contradicting collective classification schemes had to be reconciled. Looking at the development of collective storage convention one sees that first of all the system of the typing pool was applied. Contrary to the operative section, in the beginning of the project, the typing pool had already experiences with the electronic storage of documents, and thus, could present a viable solution for the problem. At that time the members of the operating section were not yet aware of the implications resulting from this decision. After producing an increasing amount of electronic documents and getting used to computer based work, the members of the section got sensitive to this problem. Being in the stronger position within the organization, they were able to modify the established conventions according to their perceived requirements. Nevertheless even at this point the section manager had still problems to anticipate his particular interests which were different from the other members of the section. Nevertheless, in the following he was able to renegotiate and modify the existing conventions for storing documents.

On the other hand we were facing the problem on how to reconcile different individual preferences. The research on the document organization on individual desks has revealed already a big variety of different storing schemes. These individual differences threatened the implementation of the storing conventions agreed upon in the workshops. Indeed the compromise for organising the shared workspace was just put into practice by those members of the section who felt that an additional level within the folder hierarchy would be helpful for them. Thus, beyond the problem of finding appropriate conventions for location based storing there is the other problem on how to motivate individuals to put these agreements into practice.

To avoid such problems, Malone (1983) has suggested to simply allow multiple classification schemes. Indeed LinkWorks provided functionality to create multiple folder hierarchies. It allowed to generate different aliases for one document. By organising the aliases in the individually created folder-hierarchy each user could realize his own view on the shared workspace (cf. Mark and Prinz 1997). In this way a shared view realising the negotiated convention and an arbitrary amount of individual views could have been created. Nevertheless the user did not make use of this feature. They pointed out that the maintenance of their individual folder hierarchy was too troublesome and labourintensive because they had to create and store aliases for every newly created document. By contrary, multiple classification schemes which were provided by different display options for documents within one folder were 
highly appreciated by the users because the different schemes could be easily generated. Thus, the experiences reported on indicate that as long as the individual organization of documents has to be performed manually, one should try to establish a common classification scheme for location based storing in a shared workspace.

Looking at this negotiation process, it turns out that experiences concerning the storage of electronic documents and the power-position of the different collective and individual stakeholders influence its outcome considerably. Moreover, the facilitation of members of the project-team played an important role in the negotiation process. As the members of the project team had a rather profound knowledge about the work practice of the different users and a clear idea of the options the system's functionality offered, they were able to suggest possible approaches to solutions. Though facilitators disposing of technical and organizational knowledge might ease negotiations, the establishment of a classification scheme to store documents in a shared workspace has to be seen as an evolutionary process. This is due to the fact that individual users and groups of users do not perceive their requirements immediately and that new technical search and display functions might effect the users' requirements for a classification scheme. Moreover, if the participants in electronicclly supported work processes change, changes of collective classification schemes might be required. In our case a widening of the field of application within the ministry which will introduce electronic document handling within the registrar and within further levels of the ministry's hierarchy is likely to affect the storing scheme.

Let's look at the reminding function of a document's allocation. The case study reveals that besides the organization of documents on a desktop the reception of paper documents can serve as a reminder to carry out tasks with them. Working in a shared workspace means to loose the reminding function of the document reception. Therefore, the users asked for a compensation by an additional functionality, the event service.

As a sophisticated event service was not foreseen in the design of the LinkWorks system, we could not implement it in the POLITeam II version. A more tailorable design of the base system would have eased to satisfy the users' requirements at this point (cf. Henderson and Kyng 1991). Looking at the benefits the users got from the quick implementation of the new display function, it becomes obvious that evolutionary negotiation processes on storage and retrieval conventions benefit strongly from a tailorable design.

\section{REFERENCES}

Barreau, D. K.: Context as a Factor in Personal Information Management Systems, in: Journal of the American Society of Information Science, Vol. 45, No. 5, 1995, pp. 327 339

Barreau, D. K.; Nardi, B. A.: Finding and Reminding - File Organization from the Desktop, in: SIGCHI Bulletin, Vol. 27, No. 2, 1995, pp. $39-43$

Henderson, A.; Kyng, M.: There's No Place Like Home: Continuing Design in Use; in: J. Greenbaum; M. Kyng (eds): Design at Work - Cooperative Design of Computer Artifacts, Hillsdale, 1991, pp. 219 - 240

Kahler, H.: Developing Groupware with Evolution and Participation: A Case Study, in: Proceedings of the Forth Biennial Conference on Participatory Design (PDC'96), Boston, MA, Nov. 13 - 15, 1996, pp. 173 - 182

Klöckner, K.; Mambrey, P.; Sohlenkamp, M.; Prinz, W.; Fuchs, L. Kolvenbach, S.; Pankoke-Babatz, U.; Syri, A.: POLITeam - Bridging the Gap between Bonn and Berlin for and with the Users, in: H. Marmolin; Y. Sundblad; K. Schmidt (eds): Proceedings of the ECSCW'95 Conference, Dordrecht, 1995, pp. $17-32$

Kwasnik, B. H.: The Influence of Context on Classification Behavior, Doctoral thesis, Rutgers University, 1989

Malone, T. W.: How do People organize their Desks?, in: ACM Transactions on Office Information Systems, Vol. 1, No. 1, 1983, pp. $99-112$

Mambrey, P.; Mark, G.; Pankoke-Babatz, U.: Integrating User Advocacy into Participative Design in: Proceedings of the Forth Biennial Conference on Participatory Design (PDC'96), Boston, MA, Nov. 13 - 15 1996, pp. 251 - 260

Mark, G; Prinz, W.: The Establishment and Support of Conventions for Groupware, in this volume

Nardi, B.; Anderson, K.; Erickson, T.: Filing and Finding Computer Files. Technical Report \# 118, Cupertine: Apple Computer, Inc. 1994

Rao, R.; Card, S.; Johnson, W.; Klotz, L.; Trigg, R.: Protofoil: Storing and Finding the Information Worker's Paper Documents in an Electronic File Cabinet, in: Proceedings of the CHI'94, Boston, MA, April 24-28, ACM-Press, New York 1994, pp. 180 - 185

Suchman, L.; Wynn, E.: Procedures and Problems in the Office, in: Office Technology and People, Vol. 2, 1984, pp. $133-154$

Wulf, V.: On Conflicts and Negotiation in Multi-User Applications; in: Kent, A.: Williams, J. G. (eds): Encyclopedia of Microcomputers, Dekker, New York et al., 1997 (in press) 